\title{
Postprandial hormone and metabolic responses amongst shift workers in Antarctica
}

\author{
J Lund', J Arendt, S M Hampton, J English and L M Morgan
}

School of Biomedical and Life Sciences, University of Surrey, Guildford, Surrey GU2 7XH, UK

${ }^{1}$ British Antarctic Survey, High Cross, Madingley Road, Cambridge, UK

(Requests for offprints should be addressed to L M Morgan; Email: I.morgan@surrey.ac.uk)

\begin{abstract}
The circadian rhythms of many night-shift workers are maladapted to their imposed behavioural schedule, and this factor may be implicated in the increased occurrence of cardiovascular disease (CVD) reported in shift workers. One way in which CVD risk could be mediated is through inappropriate hormonal and metabolic responses to meals. This study investigated the responses to standard meals at different circadian times in a group of night-shift workers on a British Antarctic Survey station at Halley Bay $\left(75^{\circ} \mathrm{S}\right)$ in Antarctica.

Twelve healthy subjects (ten men and two women) were recruited. Their postprandial hormone and metabolic responses to an identical mixed test meal of $3330 \mathrm{~kJ}$ were measured on three occasions: (i) during daytime on a normal working day, (ii) during night-time at the beginning of a period of night-shift work, and (iii) during the daytime on return from nightworking to daytime working. Venous blood was taken for $9 \mathrm{~h}$ after the meal for the measurement of glucose, insulin, triacylglycerol (TAG) and non-esterified fatty acids. Urine was collected 4-hourly (longer during sleep) on each test day for assessment of the circadian phase via 6sulphatoxymelatonin (aMT6s) assay.

During normal daytime working, aMT6s acrophase was delayed $(7 \cdot 7 \pm 1 \cdot 0 \mathrm{~h}$ (s.E.M.)) compared with that
\end{abstract}

previously found in temperate zones in a comparable age-group. During the night shift a further delay was evident $(11.8 \pm 1.9 \mathrm{~h})$ and subjects' acrophases remained delayed 2 days after return to daytime working $(12 \cdot 4 \pm 1 \cdot 8 \mathrm{~h})$. Integrated postprandial glucose, insulin and TAG responses were significantly elevated during the night shift compared with normal daytime working. Two days after their return to daytime working, subjects' postprandial glucose and insulin responses had returned to pre-shift levels; however, integrated TAG levels remained significantly elevated.

These results are very similar to those previously found in simulated night-shift conditions; it is the first time such changes have been reported in real shift workers in field conditions. They provide evidence that the abnormal metabolic responses to meals taken at night during unadapted night shifts are due, at least in part, to a relative insulin resistance, which could contribute to the documented cardiovascular morbidity associated with shift work. When applied to the $20 \%$ of the UK workforce currently employed on shift work, these findings have major significance from an occupational health perspective.

Journal of Endocrinology (2001) 171, 557-564

\section{Introduction}

Shift work has become a way of life for approximately $20 \%$ of the work force in the UK. Health problems within this population are therefore of economic and industrial importance. An increased incidence of cardiovascular disease (CVD) is well documented (Knutsson et al. 1986, Kristensen 1989, Kawachi et al. 1996). In the Helsinki heart study the relative risk of CVD amongst shift workers compared with dayworkers was 1.4 after adjustment for lifestyle factors, blood pressure and fasting lipid levels (Tenkanen et al. 1997). The causes are likely to be multifactorial; however, a major factor is considered to be the maladaptation of endogenous circadian rhythms to abrupt changes in shift times. It has been shown, under experimental conditions, that following an abrupt change in the timing of sleep and work, postprandial glucose and lipid tolerance and insulin secretion are significantly altered, to an extent that may contribute to atherogenic sequelae and hence CVD (Hampton et al. 1996, Laakso 1996).

Glucose tolerance is known to decrease during the day in normal individuals (Carroll \& Nestel 1973). Studies have been carried out to investigate postprandial plasma glucose levels following bolus doses of glucose at different times of the day (Service et al. 1983). The persistence of 
the diurnal variation of glucose tolerance under conditions devoid of zeitgebers (the environmental factors that entrain or synchronise a rhythm) strongly suggests that the normal pattern of glucose tolerance, through a given $24 \mathrm{~h}$ period, must at least be partially controlled by signals originating from the body clock (Van Cauter et al. 1989). Studies investigating the effects of time of day on glucose tolerance have shown both glucose and insulin responses to be modulated by circadian rhythmicity (Van Cauter et al. 1992). The mechanisms for these time-dependent changes in glucose tolerance are beginning to be elucidated with both diminished insulin sensitivity and decreased insulin secretion being implicated (Carroll \& Nestel 1973, Van Cauter et al. 1989, 1991).

Numerous factors are known to influence postprandial circulating lipid levels. The rates of gastric emptying, intestinal triacylglycerol (TAG) hydrolysis and intestinal motility all contribute to the rate of TAG entry into the circulation (Cohn et al. 1989). Lipoprotein lipase, the activity of which is influenced by insulin, plays a key regulatory role in postprandial TAG clearance (Knapper et al. 1995). Higher postprandial plasma TAG levels have been reported following meals at night compared with during the day (Romon et al. 1997). Non-esterified fatty acids (NEFAs) show a diurnal rhythm, although this appears to be largely related to meal patterns (Frayn et al. 1996). Circulating NEFA levels are, however, also influenced by the action of insulin in inhibiting hormonesensitive lipase.

We have previously shown that immediately after a $9 \mathrm{~h}$ phase advance, such as might be found on rotating shift work, postprandial glucose, insulin and TAG responses to a standard mixed test meal are significantly greater than to the same meal at the same external clock time before the phase shift (Hampton et al. 1996, Ribeiro et al. 1998). Thus each time a dayworker starts a night shift or an adapted nightworker starts a day shift, they are likely to show hormonal and metabolic abnormalities. In studies of simulated shift work in a controlled laboratory environment, we have confirmed the occurrence of greater postprandial hormone and metabolic responses at night in unadapted individuals (Ribeiro et al. 1999).

The aim of the present study was to extend these observations on postprandial hormone and metabolic responses during simulated shift work to a real-life shiftworking environment. We have previously shown that night-shift workers in Antarctica usually show complete circadian adaptation to night-shift work (0000-0800 h) within 7 days, comparable with oil rig staff working 1800-0600 h for 2 weeks (Midwinter \& Arendt 1991, Ross et al. 1995, Barnes et al. 1998). A group of night-shift workers on a British Antarctic Survey station in Antarctica (Halley Bay, $75^{\circ} \mathrm{S}, 20^{\circ} \mathrm{W}$ ) were investigated. They provide an interesting and relatively controlled population for investigation of responses to standard meals at different circadian times. This study describes the working pattern and change in circadian phase around a change from daywork to nightwork and upon the return to dayworking. The postprandial hormone and metabolic responses to three standard mixed test meals, given (i) during the daytime of a normal working day, (ii) at night-time at the beginning of a period of night-shift work, and (iii) during the daytime on return to dayworking following a period of night-shift work, have been measured.

\section{Materials and Methods}

\section{Subjects}

Twelve healthy subjects, two women and ten men, aged 24-34 years (mean \pm s.E.M. $28 \pm 1 \cdot 9$ years) and weighing between 60 and $88 \mathrm{~kg}(74.3 \pm 6.5 \mathrm{~kg})$ were recruited from the over-wintering population of scientists and support workers at Halley Bay Research Station, Coats Land, Antarctica. Each over-winterer at Halley works one or two night shifts in rotation during the winter, a period of approximately 20 weeks. Subjects refrained from smoking (if smokers, $n=3$ ), over the period of their study meals, took no medication and also refrained from heavy exercise and alcohol, again for the period of study. Nightwork consisted of a fire watch, with additional duties including the recording of meteorological information and baking bread for the remaining members of the base complement. Subjects were encouraged to pursue their own activities, within the remit of the study, when not conducting their rounds or performing the other required duties during this 7 day working week. All subjects were screened medically prior to the commencement of their Antarctic tours and no intercurrent medical condition was reported to the base doctor by any of the subjects over the study period. Consent was obtained from all subjects after full explanation of the purpose and nature of all procedures used. The study was approved by the University of Surrey Advisory Committee on Ethics, functioning according to the guidelines issued by the Royal College of Physicians of London in August 1996.

\section{Shift-working protocol}

Subjects worked a normal 'nine-to-five' pattern for the week prior to the night shift, and then abruptly changed to working nights from $0000 \mathrm{~h}$ to $0800 \mathrm{~h}$ on the Friday of the last week of days. The week of night shifts was worked and a similar abrupt shift in phase was experienced after seven nights for the return to normal daytime work. The shifts are illustrated in Fig. 1. The shaded areas represent the $8 \mathrm{~h}$ period normally considered available for sleep; regular sleep/wake cycles were, however, not imposed on the subjects. All 12 subjects completed their duties on night watch, staying awake throughout the required work 
Time of Day (h)

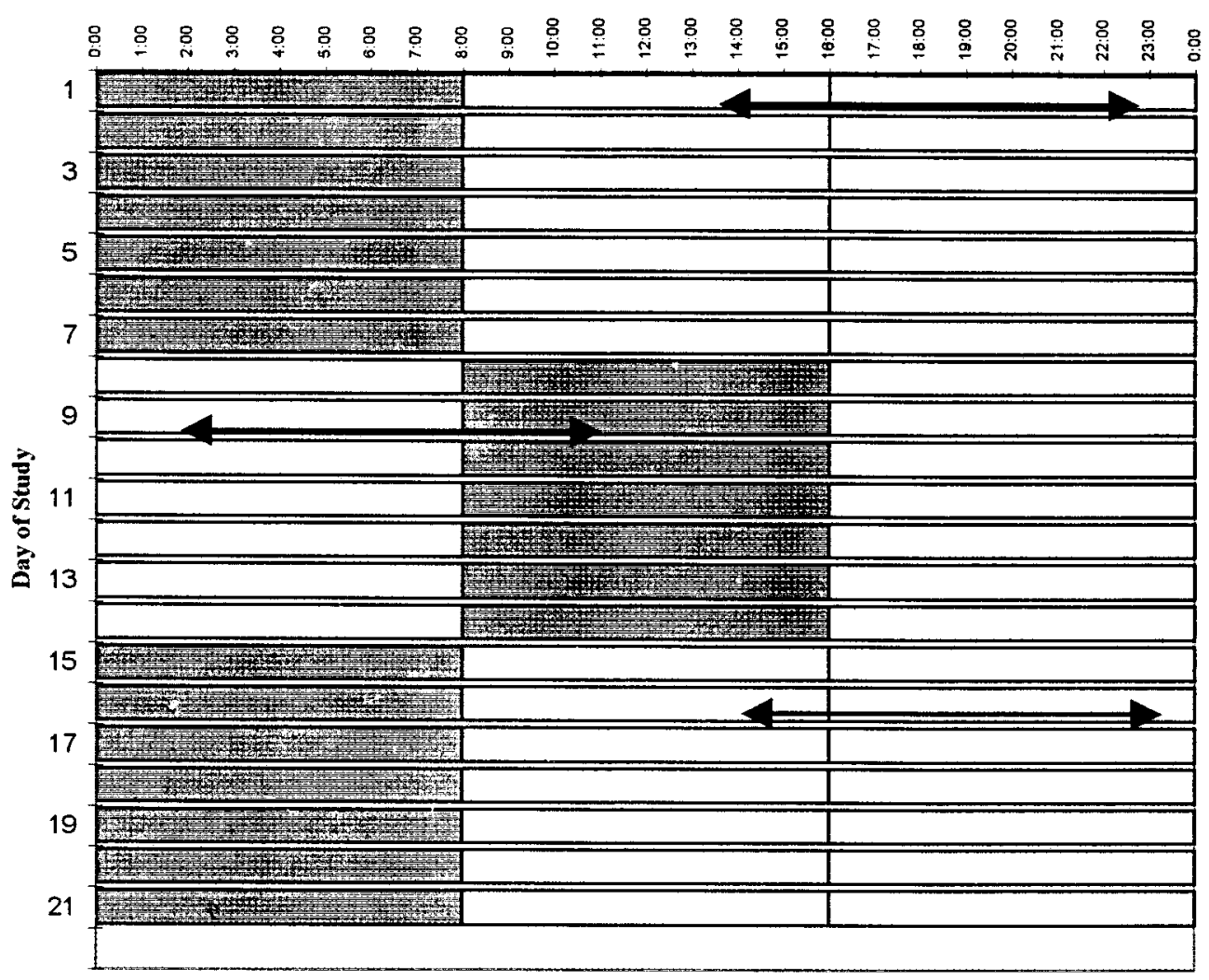

Figure 1 Diagrammatic representation of shift-work protocols. Shaded areas denote an 8-h period normally considered available for sleep. $\leftrightarrow$ Denotes postprandial test meal study. Day $1=$ normal daytime working, day $9=$ commencement of night shift, day $16=$ return to day-shift working.

period and, as would be expected from any working population, they took sufficient rest and sleep during their free time.

The phase shift was monitored using the circadian rhythm marker, urinary 6-sulphatoxymelatonin (aMT6s). Urine was collected every $4 \mathrm{~h}$ (every $8 \mathrm{~h}$ when the subjects were asleep) on two baseline days and the three test meal study days for measurement of the major melatonin metabolite, aMT6s. The volume was recorded and aliquots were frozen at $-20{ }^{\circ} \mathrm{C}$ for shipment back to the UK and subsequent analysis.

\section{Test meal studies}

Subjects were studied three times: on day 1 (during the daytime of their normal working day, within 2 weeks of commencing the night shift), day 9 (at night-time on the second night of the night shift) and day 16 (during the daytime on the second day of return to dayworking). Days 9 and 16 were chosen to minimise any effect of sleep debt around shift changeover, whilst retaining maladaptation following an abrupt shift change. At $0830 \mathrm{~h}$ on each of the study days (2030 h on day 9), subjects were given a set pre-meal $(2066 \mathrm{~kJ}, 27 \cdot 5 \%$ fat, $8 \cdot 4 \%$ protein and $64 \cdot 1 \%$ carbohydrate). Subjects then refrained from eating or drinking until the test meal, apart from water, which was freely available throughout the entire study. At $1330 \mathrm{~h}$ $(0130 \mathrm{~h}$ on day 9), subjects were given a test meal comprising $3330 \mathrm{~kJ}, 37 \%$ fat, 52\% carbohydrate and 11\% protein. All meals were consumed within $15 \mathrm{~min}$. An indwelling cannula was placed in an antecubital vein prior to starting the test meals. Two baseline blood samples (at -10 and $0 \mathrm{~min}$ ) were taken and sampling was continued for $9 \mathrm{~h}$ after consumption of the test meal $(15,30,45,60,90,120,180,240,300,360,420,480$ and $540 \mathrm{~min}$ ). Plasma was separated immediately by centrifugation, aliquoted and stored at $-20{ }^{\circ} \mathrm{C}$ before ultimately being shipped back to the UK for analysis.

\section{Assay procedures}

Plasma glucose and TAG (reagents for both from Roche Products Ltd, Welwyn Garden City, Herts, UK) and NEFAs (Wako Chemicals, Neuss, Germany) were 


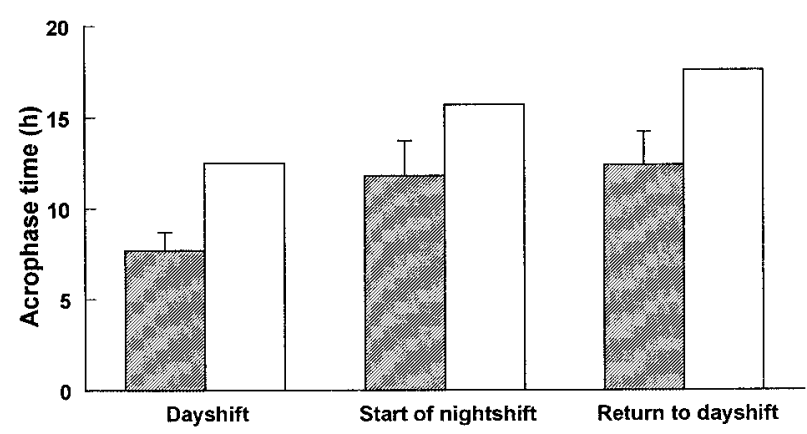

Figure 2 Urinary aMT6s acrophase (calculated peak) in healthy Antarctic shift workers, during normal daytime working (day 1 ), commencement of the night shift (day 9) and return to day-shift working (day 16). Shaded bars are means \pm S.E.M. for the entire group, $n=12$; open bars are means for delayed subjects, $n=3$.

measured by standard automated enzymatic spectrophotometric methods. The interassay coefficients of variation were less than $5 \%$ for these assays. Plasma immunoreactive insulin (Hampton \& Withey 1993) and aMT6s (Arendt et al. 1985, modified by Aldous \& Arendt 1988) were measured using in-house RIAs. The interassay coefficients of variation were less than $10 \%$ for these assays. For all analyses, samples obtained following the test meals, before and after phase shift, from a single subject were measured in the same assay.

\section{Data and statistical analysis}

Urine aMT6s data underwent a computerised cosinor analysis, using a program developed by Dr D S Minors at the University of Manchester, UK, to ascertain calculated peak times of melatonin secretion (acrophase). The program fits a sine wave to the data and derives the mathematical peak time, together with the percentage of variance accounted for by the sine wave. The derived peak times correlate very closely with those calculated from hourly measures of plasma melatonin $(r>0 \cdot 9$, Ross et al. 1995), the best marker of circadian phase (i.e. the timing of the internal circadian clock).

Postprandial hormone and metabolite responses were estimated as the total area under the response curve (AUC), calculated using the trapezoidal rule.

All hormonal and metabolic data were compared by repeated-measures ANOVA, followed by the TukeyKramer multiple comparisons test to locate individual differences, using the Instat statistical package (Graphpad, San Diego, CA, USA).

\section{Results}

Circadian rhythm markers

Urinary aMT6s acrophase data are shown in Fig. 2. Repeated-measures ANOVA for all subjects revealed a significant effect of shift working on subjects' urinary aMT6s acrophase (peak) times $(P<0 \cdot 05)$. On day 1 (during normal daytime working) subjects' mean acrophase was $7 \cdot 7 \pm 1 \cdot 0 \mathrm{~h}$. A non-significant delay in mean acrophase had occurred by the second day of night shifts (day 9, acrophase $11.8 \pm 1.9 \mathrm{~h}$ ). On the second day of return to daytime working following night shifts, subjects' acrophases remained delayed; they were now significantly different from those observed during normal daytime working (day 1 vs day $16, P<0 \cdot 05$ ). Three of the 12 subjects had very delayed acrophase times during normal daytime work, which were comparable with the whole group's mean levels on night shifts. However, these subjects' acrophases delayed still further during the night shift, in parallel with the rest of the group (Fig. 2) and all the subjects were therefore included in all subsequent analyses.

\section{Fasting and postprandial hormone and metabolite responses}

Fasting plasma levels of glucose, insulin and NEFA were similar for all study days. Repeated-measures ANOVA demonstrated differences in fasting TAG levels between the three study days $(P<0 \cdot 05)$ with a trend towards higher mean fasting TAG levels on return to dayworking following night shifts, compared with normal daytime working (mean fasting TAG day $1,1.4 \pm 0.2 \mathrm{mmol} / \mathrm{l}$; day 16 , $1 \cdot 8 \pm 0 \cdot 2 \mathrm{mmol} / 1, P=0 \cdot 07)$.

Postprandial glucose and insulin data are shown in Fig. 3. Postprandial glucose tolerance, as assessed by the postprandial glucose AUC, deteriorated significantly at the commencement of night-shift work (day 1 vs day 9, $P<0 \cdot 01)$. However, it improved on return to daywork and on day 16 was indistinguishable from levels achieved during normal daytime working (day 1 vs day 16). Patterns of plasma insulin were similar to those for glucose. Plasma insulin levels were significantly greater on the second night of night shifts $(P<0 \cdot 01)$ compared with normal daytime working, but they had returned to normal daytime working patterns 2 days from the return from night-shift to daytime working (day 16).

Postprandial TAG and NEFA data are shown in Fig. 4. The postprandial TAG AUC was significantly greater on the second day of night-shift work compared with normal daywork (day 1 vs day $9, P<0 \cdot 05$ ) and it remained elevated 2 days following the return to daytime working (day 1 vs day 16, $P<0 \cdot 01$ ). Postprandial NEFA data mirrored those of TAG. The plasma NEFA AUC was significantly smaller on night shifts compared with normal daytime working (day 1 vs day $9, P<0 \cdot 01$ ) and it remained smaller on return to daytime working (day 1 vs day 16 , $P<0 \cdot 01)$.

\section{Discussion}

Acrophase data from the urinary circadian rhythm marker aMT6s demonstrated that subjects in this study were 


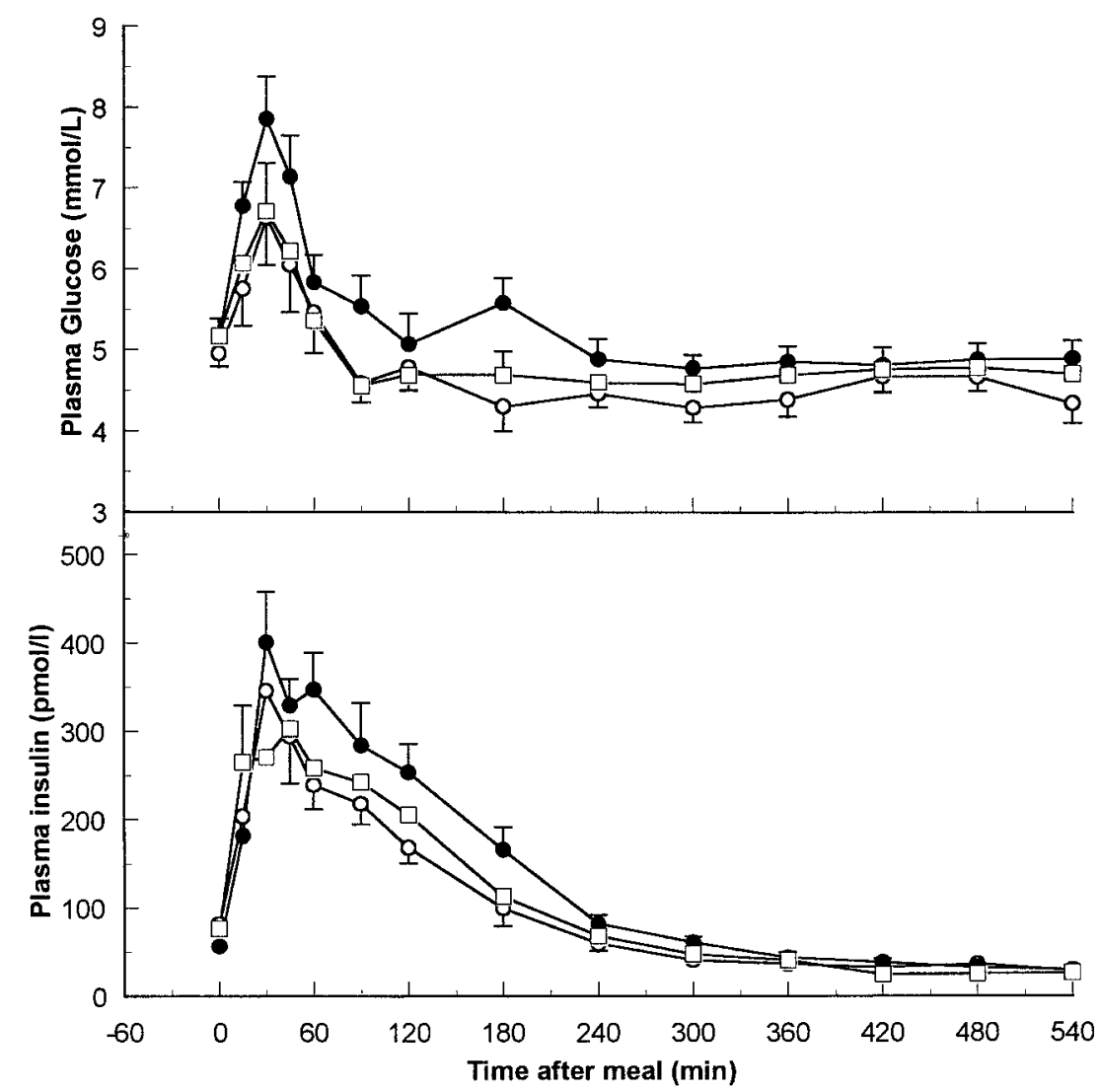

Figure 3 Postprandial plasma glucose and insulin levels following a $3330 \mathrm{~kJ}$ test meal in healthy Antarctic shift workers. $\bigcirc$ Day 1 (normal daytime working), - day 9 (at commencement of the night shift), $\square$ day 16 (immediate return to daytime working). Means \pm S.E.M., $n=12$. Integrated postprandial glucose and insulin responses for $0-540 \mathrm{~min}$, day 9 , were significantly different from day $1(P<0 \cdot 01)$ and day $16(P<0 \cdot 05)$ by

Tukey-Kramer multiple comparison test.

phase-delayed compared with those of a comparable age-group living in temperate zones (temperate zone aMT6s typical acrophase $4 \cdot 41 \pm 1 \cdot 19 \mathrm{~h}$, mean \pm s.D., $n=52$ ). This is consistent with previous observations in over-wintering Antarctic staff (Ross et al. 1995). Also consistent with previous findings are the data for the return meal (day 16), where there has failed to be a significant shift in phase back towards that of daytime living. Readaptation following shift work in an Antarctic environment, particularly during the winter period where base members do not experience light intensities greater than $500 \mathrm{~lx}$ for a period of at least 4 months, has been found to take up to 3 weeks without the use of bright light to help resynchronisation (Midwinter \& Arendt 1991). Similar observations using behavioural measures have been made on North Sea oil rigs (Bjorvatn et al. 1999).

Since many circadian rhythms shift in parallel with the melatonin/aMT6s rhythm, it is a reasonable assumption that, for example, cortisol, thyrotrophin and core body temperature would be similarly shifted. The measurement of other hormones such as cortisol in these field conditions is impracticable and gives a less than accurate estimate of circadian phase. This is due to the 'masking' effects of stress and meals, and to the episodic nature of secretion, which requires frequent sampling for correct definition.

This study wished to deduce the metabolic effects of night-shift work without any contributing factors towards circadian adaptation, thus no bright light was used after the week of nights to aid resynchronisation. As a consequence, subjects' circadian rhythms were maladapted to their sleep/wake cycles and working environment both at the beginning of the night shifts (day 9) and upon return to normal daytime working (day 16). The significance of a delayed acrophase compared with subjects living in temperate zones, with respect to metabolic rhythms, is not known, but any effects are likely to be small in comparison with those induced by night-shift work.

On the second night of night-shift working, before subjects had adapted to the enforced phase-shift imposed by night-time working, all the postprandial hormone and 


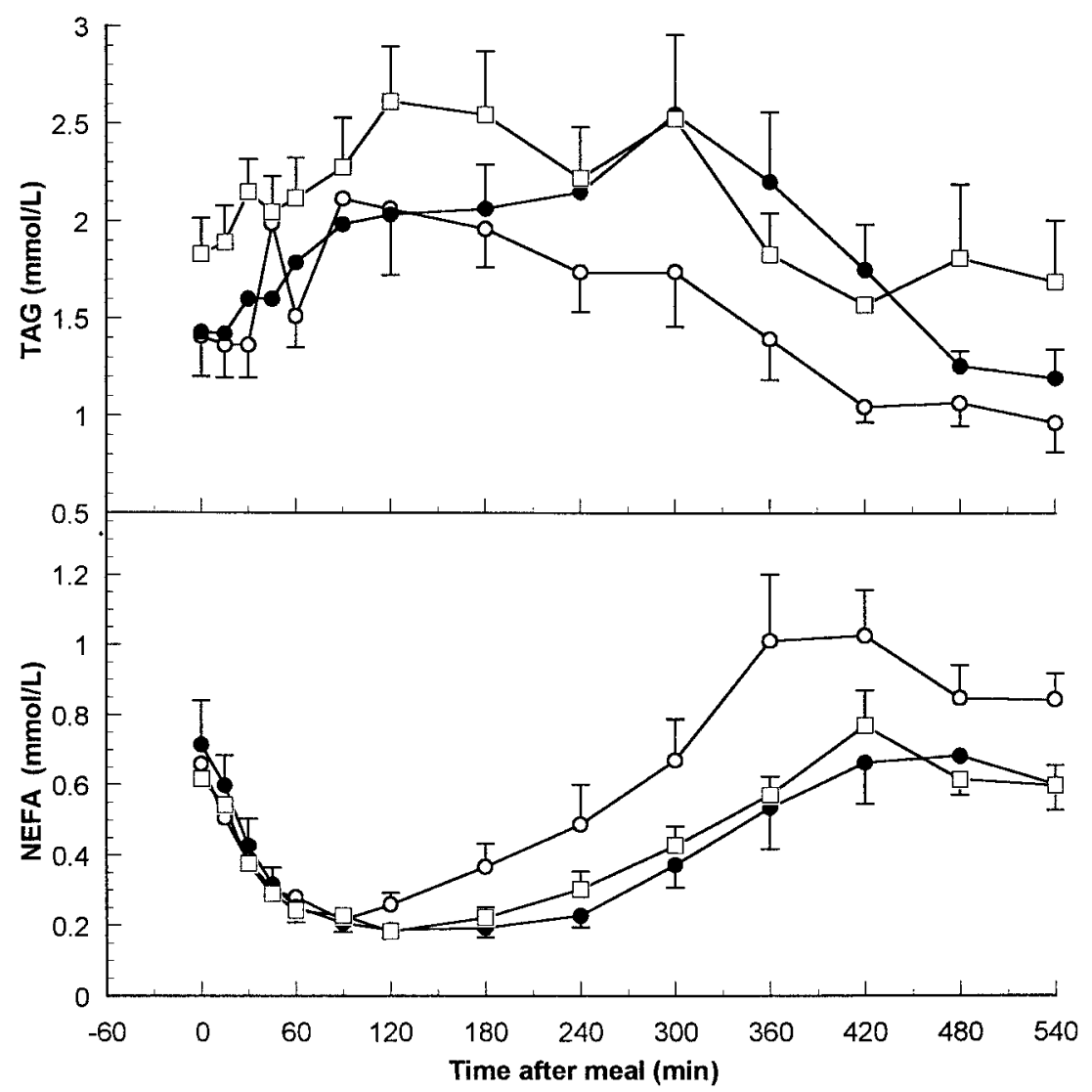

Figure 4 Postprandial plasma TAG and NEFA levels following a $3330 \mathrm{~kJ}$ test meal in healthy Antarctic shift workers. $\bigcirc$ Day 1 (normal daytime working), day 9 (at commencement of the night shift), $\square$ day 16 (immediate return to daytime working). Means \pm S.E.M., $n=12$. Integrated postprandial TAG responses for 0-540 min, day 1 , were significantly different from day $9(P<0 \cdot 05)$ and day $16(P<0 \cdot 01)$ and integrated NEFA responses for day 1 were significantly different from days 9 and $16(P<0 \cdot 01)$ by Tukey-Kramer multiple comparison test.

metabolite responses measured were altered relative to those found during normal daytime working. We have previously reported altered postprandial hormone and metabolic responses in laboratory-simulated shift-working conditions (Ribeiro et al. 1999); this is the first time such changes have been reported in real shift workers in field conditions. Both the circadian clock and the timing of sleep itself are likely to contribute to these effects (Morgan et al. 1998).

Glucose and insulin levels were both elevated during the night-shift period. This is in agreement with previous reports of impaired glucose tolerance and increased insulin secretion following night-time meals (Van Cauter et al. 1992), following a forced phase advance (Hampton et al. 1996) or in simulated night-shift workers (Ribeiro et al. 1999). These effects are due, at least in part, to a relative insulin insensitivity at night; reduced sensitivity of the peripheral tissues to insulin in the evening compared with the morning has recently been reported (Morgan et al. 1999). Postprandial plasma TAG levels were also elevated during the night-shift period relative to normal daytime working. This pattern, which has previously been found at night (Romon et al. 1997), during forced phase advance (Ribeiro et al. 1998) and during simulated nightshift working (Ribeiro et al. 1999), is also suggestive of insulin resistance. The magnitude of these changes at night appears to be influenced by the preceding diet; we have previously shown that a very low fat (4\% of total energy) meal prior to the test meal can prevent the deterioration of glucose, but not lipid, tolerance following forced circadian phase advance (Ribeiro et al. 1998). In the present study, the subjects' previous meal was relatively low in fat $(27.5 \%$ of total energy as fat, which compares with average UK daily fat intakes of approximately 38\%). Nevertheless, changes in postprandial glucose and insulin, as well as TAG, were observed during the night shift. Plasma NEFA levels were suppressed postprandially; integrated levels were lower and took longer to return to basal during the 
night shift. Circulating NEFA is partly derived form the hydrolysis of adipose tissue TAG by hormone-sensitive lipase. This enzyme is very sensitive to inhibition by insulin, and the lower NEFA levels are likely to be a reflection of the higher postprandial insulin levels observed during the night shift.

We have previously shown that night-shift workers in Antarctica usually show complete circadian adaptation to night-shift work within 7 days (Ross et al. 1995). Two days post-night shift, subjects had failed to readapt back completely to normal daytime rhythms. At this time, subjects' postprandial TAG levels remained significantly elevated; however, their glucose and insulin levels had returned to pre-shift working levels. In a 'constant routine' study, designed to dissociate internal circadian effects from other factors, we have previously shown postprandial TAG and glucose responses to be significantly affected by both the endogenous circadian 'clock time' and the length of subject's prior wakefulness, whereas insulin is affected only by endogenous clock time (Morgan et al. 1998). During the night-shift test meal, subjects had remained awake for longer than for either of the daytime meals (Fig. 1), which may provide some explanation of the divergence of TAG from the glucose and insulin data on return to daytime working. Glucose tolerance declines with increasing sleep debt (Spiegel et al. 1999), and we suggest that the rapid return to normal values is a function of time awake prior to the meal overriding any clock-related changes. It is, however, of interest that subjects at Halley base, in contrast to the majority of other shift workers, have previously reported an improvement in sleep during the night shift (Ross et al. 1995)

Both insulin resistance and elevated postprandial plasma TAG levels are independent risk factors for CVD (Hokanson \& Austin 1996, Lyons \& Jenkins 1997, Reaven 1998). TAG is a higher independent risk factor for CVD in women than in men (Austin 1997) and a recent study has shown myocardial infarction risk to be associated with shift work with a relative risk of 1.6 for men and 3.0 for women in the age-group 45-55 (Knutsson et al. 1999). Circulating TAG levels in excess of $1.5 \mathrm{mmol} / 1$ predispose to the formation of atherogenic small dense low-density lipoproteins (Griffin 1999). In our study, mean TAG levels exceeded this threshold immediately following night-shift working, both when fasting and for the entire postprandial period. The results of the present study agree with those of previous experiments simulating a rotating shiftwork pattern. They suggest that night-shift workers may develop significant abnormalities in postprandial hormone and metabolic responses after abrupt phase shifts, demonstrating insulin insensitivity and lipid intolerance. In the present study, these effects had not fully resolved 2 days after returning to normal daytime working. Shift scheduling determines the degree and direction of circadian adaptation to the night shift (Barnes et al. 1998). A similar adaptation to that seen in Antarctica is present during the commonly used night-shift schedule working 18000600 h on North Sea oil rigs (Barnes et al. 1998). It is likely that not only lack of daylight, but also absence of normal social activities and quiet, dark sleeping quarters, enable adaptation to occur; it is uncommon to see adaptation in temperate zones onshore.

Our study has demonstrated that maladaptation of endogenous circadian rhythms to the abrupt changes in shift times is associated with metabolic sequelae of insulin resistance; this could play a role in the documented cardiovascular morbidity associated with shift work. When applied to $20 \%$ of the country's workforce currently employed on shift working, these findings have major significance from an occupational health perspective. Further studies are needed to provide the level of information required to optimise shift-working schedules for long-term health.

\section{References}

Aldous M \& Arendt J 1988 Radioimmunoassay for 6-sulphatoxymelatonin in urine using an iodinated tracer. Annals of Clinical Biochemistry 25 298-303.

Arendt J, Bojkowski C, Franey C, Wright J \& Marks V 1985 Immunoassay of 6-hydroxy-melatonin sulphate (aMT6s) in human plasma and urine: abolition of the urinary 24-hour rhythm with atenolol. Journal of Clinical Endocrinology and Metabolism $\mathbf{6 0}$ 1166-1173.

Austin MA 1997 Triacylglycerol and coronary heart disease. Proceedings of the Nutrition Society $\mathbf{5 6} 667-670$.

Barnes RG, Forbes MJ \& Arendt J 1998 Shift type and season affect adaptation of the 6-sulphatoxymelatonin rhythm in offshore oil rig workers. Neuroscience Letters 252 179-182.

Bjorvatn B, Kecklund G \& Akerstedt TJ 1999 Bright light treatment used for adaptation to night work and re-adaptation back to day life. A field study at an oil platform in the North Sea. Sleep Research 2 105-120.

Carroll KF \& Nestel PJ 1973 Diurnal variation in glucose tolerance and in insulin secretion in man. Diabetes 22 333-348.

Cohn J, McNamara J, Krasinski S, Russel R \& Scheefer E 1989 Role of triglyceride rich lipoproteins from the liver and intestines in the aetiology of postprandial peaks in plasma triglycerides. Metabolism $\mathbf{3 8}$ 484-490.

Frayn KN, Williams CM \& Arner P 1996 Are increased plasma nonesterified fatty acid concentrations a risk marker for coronary heart disease and other chronic diseases? Clinical Science 90 243-253.

Griffin BA 1999 Lipoprotein atherogenicity: an overview of current mechanisms. Proceedings of the Nutrition Society 58 163-169.

Hampton SM \& Withey L 1993 Monitoring B-cell responses in obese and normal weight subjects; a pilot study. Diabetes and Metabolism $19582-585$.

Hampton SM, Morgan LM, Lawrence N, Anastasiadou T, Norris F, Deacon S \& Arendt J 1996 Postprandial hormone and metabolic responses in simulated shift work. Journal of Endocrinology 151 259-267.

Hokanson JE \& Austin MA 1996 Plasma triglyceride levels as an independent risk factor for cardiovascular disease: a meta-analysis of population-based prospective studies. Journal of Cardiovascular Disease Risk 3 213-219.

Kawachi I, Colditz GA \& Stamfer MJ 1996 Prospective study of shift work and risk of coronary heart disease in women. Circulation 92 3178-3182.

Knapper JME, Puddicombe SM \& Morgan LM 1995 Enteroinsular hormones glucose-dependent insulinotropic polypeptide and 
glucagon-like peptide-1 (7-36)amide; effects on lipoprotein lipase activity in explants of rat adipose tissue. Journal of Nutrition $\mathbf{1 2 5}$ 183-188.

Knutsson A, Akerstedt T, Jonsson BG \& Orth-Gomer K 1986 Increased risk of ischaemic heart disease in shift workers. Lancet $\mathbf{i}$ 89-92.

Knutsson A, Hallquist J, Reuterwall C, Theorell T \& Akerstedt T 1999 Shiftwork and myocardial infarction: a case-control study. Occupational and Environmental Medicine 56 46-50.

Kristensen TS 1989 Cardiovascular diseases and the work environment. A critical review of the epidemiological literature on nonchemical factors. Scandinavian Journal of Work and Environmental Health 15 165179.

Laakso M 1996 Insulin resistance and coronary heart disease. Current Opinion in Lipidology 7 217-226.

Lyons TJ \& Jenkins AJ 1997 Lipoprotein glycation and its metabolic consequences. Current Opinion in Lipidology 8 174-180.

Midwinter MJ \& Arendt J 1991 Adaptation of the melatonin rhythm following night-shift work in Antarctica. Neuroscience Letters 122 195-198.

Morgan LM, Arendt J, Owens D, Folkard S, Hampton S, Deacon S, English J, Ribeiro D \& Taylor K 1998 Effects of the endogenous clock and sleep time on melatonin, insulin, glucose and lipid metabolism. Journal of Endocrinology 157 443-451.

Morgan LM, Apostolakou F, Wright J \& Gama R 1999 Diurnal variations in peripheral insulin resistance and plasma non-esterified fatty acid concentrations: a possible link? Annals of Clinical Biochemistry 36 447-450.

Reaven GM 1998 Role of insulin resistance in human disease. Diabetes 37 1595-1607.

Ribeiro DCO, Hampton SM, Morgan L, Deacon S \& Arendt J 1998 Altered postprandial and metabolic responses in a simulated shift work environment. Journal of Endocrinology 158 305-310.
Ribeiro DCO, Hampton SM, Morgan L \& Arendt J 1999 Inappropriate nocturnal postprandial responses amongst shift workers. Proceedings of the Nutrition Society 58 34A.

Romon M, Le Fur C, Lebel P, Edme J-L, Fruchart J-C \& Dallongeville J 1997 Circadian variation of postprandial lipaemia. American Journal of Clinical Nutrition 65 934-940.

Ross JK, Arendt J, Horme J \& Haston W 1995 Night-shift work in Antarctica: sleep characteristics and bright light treatment. Physiology and Behavior 57 1169-1174.

Service FJ, Hall LD, Westland RE, O'Brien PC, Go VLQ, Raymond MW \& Rizza RA 1983 Effects of size, time of day and sequence of meal ingestion on carbohydrate tolerance in subjects. Diabetologia 25 316-321.

Spiegel K, Leproult R \& Van Cauter E 1999 Impact of sleep debt on metabolic and endocrine function. Lancet ii 1435-1439.

Tenkanen L, Sjorblom T \& Kalimo R 1997 Shift work occupation and coronary heart disease over 6 years of follow-up in the Helsinki heart study. Scandinavian Journal of Work and Environmental Health $\mathbf{2 3}$ 257-265.

Van Cauter E, Desir D, Decoster C, Fery F \& Balosse EO 1989 Nocturnal disease of glucose tolerance during constant glucose infusion. Journal of Clinical Endocrinology and Metabolism 69 604-611.

Van Cauter E, Blackman JD, Roland D, Spire JP, Refetoff S \& Polonsky KS 1991 Modulation of glucose regulation and insulin secretion by circadian rhythmicity and sleep. Journal of Clinical Investigation 88 934-942.

Van Cauter E, Shapiro ET, Tillil H \& Polonsky KS 1992 Circadian modulation of glucose and insulin response to meals: relationship to cortisol. American Journal of Physiology 262 E467-E475.

Received 6 August 2001
Accepted 21 August 2001 